Ecología

\title{
Sobre la relación entre idoneidad del hábitat y la abundancia poblacional bajo diferentes escenarios de dispersión
}

\section{On the relationship between habitat suitability and population abundance under different dispersal scenarios}

\author{
Luis Alfredo Osorio-Olvera ${ }^{\mathrm{a}, *}$, Manuel Falconi ${ }^{\mathrm{b}}$ y Jorge Soberón ${ }^{\mathrm{c}}$ \\ ${ }^{a}$ Facultad de Ciencias, Universidad Nacional Autónoma de México, Circuito exterior s/n, Cd. Universitaria, 04510, Ciudad de México, México \\ ${ }^{\mathrm{b}}$ Departamento de Matemáticas, Facultad de Ciencias, Universidad Nacional Autónoma de México, Circuito exterior s/n, Cd. Universitaria, 04510, \\ Ciudad de México, México \\ ${ }^{\mathrm{c}}$ Biodiversity Institute, University of Kansas, Dyche Hall, 1345 Jayhawk, Boulevard, Lawrence, KS, 66045, Estados Unidos \\ Recibido el 26 de febrero de 2015; aceptado el 7 de abril de 2016 \\ Disponible en Internet el 17 de agosto de 2016
}

\begin{abstract}
Resumen
Trabajos recientes basados en modelación del nicho para estimar la distribución de las especies han aportado evidencias de que la abundancia de una especie decrece conforme aumenta la distancia al centroide del nicho climático de la especie. Esta relación empírica se ha determinado utilizando modelos correlativos que relacionan datos de presencia/ausencia de una especie con factores abióticos, bajo el supuesto de que las especies se encuentran en equilibrio con su ambiente, pero sin tomar en cuenta explícitamente procesos poblacionales. La falta de un mecanismo que relacione explícitamente procesos poblacionales con el nicho de la especie dificulta la interpretación de los resultados y la obtención de conclusiones generales. En este trabajo se explora la relación entre la idoneidad del hábitat y la abundancia bajo diferentes escenarios de dispersión, utilizando una especie virtual. Se encontró que, en un escenario sin dispersión, hay una clara correlación entre la abundancia y la idoneidad del hábitat, pero en aquellos en los que se incluye el proceso de dispersión, a medida que se incrementa la proporción de individuos que se dispersan, la correlación entre abundancia e idoneidad del hábitat disminuye. Estos resultados apoyan la idea de que existe una relación entre idoneidad del hábitat y las abundancias, sin embargo, también muestran que esta se encuentra modulada por la dispersión.

Derechos Reservados (C) 2016 Universidad Nacional Autónoma de México, Instituto de Biología. Este es un artículo de acceso abierto distribuido bajo los términos de la Licencia Creative Commons CC BY-NC-ND 4.0.
\end{abstract}

Palabras clave: Modelos de distribución de especies; Modelos híbridos; Núcleo de dispersión; Especie virtual

\begin{abstract}
Recent studies based on niche modeling to estimate the distribution of species have provided evidence that the abundance of a species decreases as the distance to the centroid of the climatic niche of the species increases. This empirical relationship is determined using correlative models that relate the presence/absence of a species with abiotic factors under the assumption that species are in equilibrium with its environment, but without taking into account population processes explicitly. The lack of a mechanism that explicitly relates population processes to the niche hinders to interpret results and obtain general conclusions. In this paper the relationship between habitat suitability and abundance under different dispersal scenarios is explored using a virtual species. A clear correlation between abundance and habitat suitability in scenarios without dispersal was found, on the other hand, this correlation decreased as the proportion of individuals who are dispersed increases. These results support the idea that there is a relationship between habitat suitability and abundances, however, they also show that this relationship is modulated by dispersal. All Rights Reserved (C 2016 Universidad Nacional Autónoma de México, Instituto de Biología. This is an open access item distributed under the Creative Commons CC License BY-NC-ND 4.0.
\end{abstract}

Keywords: Species distribution models; Hybrid models; Dispersal Kernel; Virtual species

\footnotetext{
* Autor para correspondencia.

Correo electrónico: luismurao@ comunidad.unam.mx (L.A. Osorio-Olvera).

La revisión por pares es responsabilidad de la Universidad Nacional Autónoma de México.
} 


\section{Introducción}

Entender los patrones de distribución de las especies es uno de los tópicos más importantes de la ecología (Sagarin, Gaines y Gaylord, 2006; VanDerWal, Shoo, Johnson y Williams, 2009). Los patrones de distribución están determinados por factores ecológicos, evolutivos y geográficos, que hacen que el estudio de la distribución de las especies sea un problema complejo (Cain, 1994; Gaston, 2003; Udvary, 1969). Desde el trabajo clásico de Grinnell (1917), se ha considerado que las preferencias ambientales de una especie, denominadas «nicho ecológico», determinan, a través de los parámetros demográficos de cada población, las posibilidades de que una especie colonice exitosamente una localidad. En conjunto con el nicho, parámetros demográficos como la reproducción, la mortalidad y la dispersión, determinan la dinámica metapoblacional y por ende la abundancia y la distribución a diferentes escalas espaciales (Guisan y Thuiller, 2005; Lira-Noriega, Soberón y Milller, 2013; Marion et al., 2012; Schurr et al., 2007; Thuiller et al., 2008).

La forma en la que se relaciona el nicho ecológico con la abundancia es un tema que ha llamado la atención (Siqueira, Bini, Cianciaruso, Oliveira y Trivinho-Strixino, 2009; VanDerWal et al., 2009). En términos generales, se espera que la reproducción y la sobrevivencia sean altas en localidades ubicadas al interior del nicho de una especie y por ende, se alcancen abundancias poblacionales relativamente altas, y lo contrario en localidades periféricas (de Moraes y Viveiros, 2012; Maguire, 1973; Martínez-Meyer, Díaz-Porras, Townsend y Yáñez-Arenas, 2013; Siqueira et al., 2009; VanDerWal et al., 2009). Esta idea fue sugerida por Hutchinson (1957), quien expresa la posibilidad de que exista una región «óptima» dentro del nicho, es decir, con condiciones óptimas para la sobrevivencia de la especie, y regiones con condiciones menos favorables en la periferia. Maguire (1973) es el primero en proponer explícitamente la idea de que diferentes regiones del espacio de nicho corresponden a diferentes valores de la tasa intrínseca de crecimiento y de la abundancia poblacional. Una idea derivada de lo anterior es la hipótesis del «centroide del nicho», la cual plantea que las mayores abundancias se alcanzan en el centro del nicho y conforme una población esté más alejada de este, tenderá a presentar menores abundancias (Martínez-Meyer et al., 2013).

Se ha empezado a acumular evidencia empírica de que la posición de una población en el espacio de nicho determina su abundancia (de Moraes y Viveiros, 2012; Escalante y Martínez-Meyer, 2013; Martínez-Meyer et al., 2013; Siqueira et al., 2009; Torres et al., 2012; VanDerWal, 2009; YáñezArenas, Martińez-Meyer, Mandujano y Rojas-Soto, 2012; Yáñez-Arenas, Guevara, Martínez-Meyer, Mandujano y Lobo, 2014). En estos trabajos se utilizan algoritmos de estimación de nichos para obtener una aproximación al nicho fundamental (medido como «idoneidad de hábitat»), y luego se establece una correlación entre la idoneidad de hábitat con datos de abundancia tomados en campo o de la literatura. Otra forma en la que se ha analizado la relación entre el nicho y la abundancia es identificando el centroide del nicho, calculado a partir de los valores promedio de distintas variables climáticas, y estableciendo una correlación entre la idoneidad de hábitat y la distancia a ese centroide. En ninguno de estos dos casos se incluyen explícitamente procesos poblacionales como natalidad, mortalidad o migración, etc. Por tal motivo, si bien estos procedimientos pueden revelar la existencia de una relación entre abundancia y posición en el espacio de nicho, los resultados son correlaciones que no permiten profundizar en el entendimiento de los mecanismos que determinan esta relación (Dormann, 2009; Pagel y Schurr, 2012).

Se han propuesto diversos métodos para integrar aspectos demográficos en el análisis de los patrones de distribución y abundancia. Los modelos espacio-temporales, por ejemplo, combinan medidas del nicho con parámetros demográficos como la dispersión y el crecimiento poblacional. Se les denomina modelos acoplados o híbridos y pueden dar estimaciones de abundancia en un espacio geográficamente explícito (Catterall, Cook, Marion, Butler y Hulme, 2012; Nenzén, Swab, Keith y Araújo, 2012; Pagel y Schurr, 2012). Estos modelos se han utilizado frecuentemente para estimar la distribución de las especies en escenarios de cambio climático (i.e., Franklin, 2010), y para estudiar la relación entre demografía y la dinámica del área de distribución de algunas especies invasoras (Franklin, 2010; Gallien, Münkemüller, Albert, Boulangeat y Thuiller, 2010; Jongejans et al., 2008; Marion et al., 2012; Pagel y Schurr, 2012; Thuiller et al., 2008).

Trabajos como el de Jongejans et al. (2008) utilizan un modelo híbrido que incluye mecanismos poblacionales y dispersión para estimar la dispersión de una planta, pero sin referencia alguna al concepto de nicho. A su vez, Kearny y Porter (2009) explican cómo los modelos mecanísticos basados en procesos biofísicos ecofisiológicos, se pueden utilizar para estimar la distribución de una especie, pero no muestran cómo relacionarlos con los procesos demográficos y de dispersión. Buckley et al. (2010) proponen un modelo híbrido en el cual la tasa de crecimiento es función del ambiente, sin incluir al proceso de dispersión. La relación entre abundancia y nicho se menciona en un par de líneas, pero no se analiza, y finalmente, Pagel y Schurr (2012) utilizan un modelo híbrido sin estructura de edades para analizar cómo la tasa de crecimiento, la capacidad de carga y la densidad poblacional se relacionan con la probabilidad de presencia de una especie.

El objetivo de este trabajo es analizar la relación entre la abundancia y la posición en el espacio de nicho utilizando un modelo híbrido, que incorpora la estructura de edades de la población y que integra algunos parámetros poblacionales básicos, así como el proceso de dispersión. Con esta aproximación novedosa se pretende contribuir a la teoría que estudia la relación entre abundancia poblacional y la posición en el espacio del nicho, de tal forma que permita profundizar en el entendimiento de los mecanismos que determinan esta relación.

\section{Materiales y métodos}

Para estudiar la relación entre la idoneidad del hábitat y las abundancias poblacionales bajo diferentes escenarios de dispersión se utilizó una especie virtual. Se asumió que el nicho fundamental de la especie se mide en términos de la idoneidad del hábitat, la cual está definida por una función $f(x, y)$ donde 
$x$ y $y$ son las variables ambientales que definen las condiciones donde la especie puede sobrevivir. Se considera que la tasa óptima de reproducción de la especie se obtiene en un cierto punto $(\bar{x}, \bar{y})$ del nicho fundamental de modo tal que $f(x, y)$ es menor que $f(\bar{x}, \bar{y})$ para todo $(x, y) \neq(\bar{x}, \bar{y})$.

En este trabajo se utilizó como función $f(x, y)$ a la función de densidad de probabilidad normal bivariada (ecuación 1) (Broennimann et al., 2012; Saupe et al., 2012), en donde la tasa óptima de reproducción $f(\bar{x}, \bar{y})$ se alcanza en las medias de las variables independientes $x$ y $y$. La función de distribución multinormal bivariada es la siguiente:

$$
\begin{aligned}
f(x, y)= & \frac{1}{2 \pi \sigma_{x} \sigma_{y} \sqrt{1-\rho^{2}}} \exp \left[-\frac{1}{2\left(1-\rho^{2}\right)}\left(\frac{\left(x-\mu_{x}\right)^{2}}{\sigma_{x}^{2}}\right.\right. \\
& \left.\left.+\frac{\left(y-\mu_{y}\right)^{2}}{\sigma_{y}^{2}}-\frac{2 \rho\left(x-\mu_{x}\right)\left(y-\mu_{y}\right)}{\sigma_{x} \sigma_{y}}\right)\right],
\end{aligned}
$$

donde $\rho$ es la correlación entre la variable $x$ y la variable $y ; \mu_{x}$ y $\mu_{y}$ son las medias de cada una de las variables independientes; $\sigma_{x}$ y $\sigma_{y}$ son las varianzas de ambas. Este método es equivalente a utilizar un método envolvente tipo distancia de Mahalanobis (Farber y Kadmon, 2003).

La función de la ecuación 1 es una función de densidad de probabilidad y por lo tanto esta se reescaló para que sus valores se encuentren en el intervalo cerrado [0,1]. La función de reescalamiento está dada por la expresión:

$S(x, y)=\frac{f(x, y)}{\max [f(x, y) \mid x, y \in \mathbb{R}]}$.

Dicha función de reescalamiento (ecuación 2) representa la denominada idoneidad del hábitat (Nenzén et al., 2012; Helaouet, Beaugrand y Edwards, 2013), en la que el valor de $S(x, y)=1$ ocurre en los niveles de las variables ambientales donde se alcanza la tasa máxima de crecimiento poblacional.

\section{Modelado de la dinámica espacial de crecimiento de la especie}

Para analizar la relación entre abundancia poblacional y posición en el espacio de nicho bajo diferentes escenarios de dispersión, se utilizó un modelo híbrido de dinámica discreta de la forma dada por:

$$
\begin{aligned}
N_{t+1}(\text { lon }, \text { lat })= & \mathrm{S}(x(\text { lon }, \text { lat }), y(\text { lon }, \text { lat })) \mathrm{M} N_{t}(\text { lon }, \text { lat }) \\
& +\Delta(\text { lon }, \text { lat }),
\end{aligned}
$$

donde $N_{t+1}$ es el vector que contiene las abundancias de la población en cada uno de los estadios en las coordenadas (lon, lat) al tiempo $t+1$. La matriz $\boldsymbol{M}=\left(a_{i, j}\right)$ es la matriz de proyección poblacional de tipo Lefkovich (Caswell, 2000) y contiene: 1), las proporciones que pasan de un estadio a otro (elementos $a_{i, j}$ con $i \neq j$ ); 2), la proporción de individuos que se mantiene en el mismo estadio (elementos de la diagonal principal) y 3), las fertilidades de los estadios (primer renglón de la matriz). Cada elemento $a_{i, j}$ es una variable aleatoria con una
Tabla 1

Matriz de proyección poblacional de Astragalus scaphoides $(\mathrm{NR}=\mathrm{no}$ reproductiva).

\begin{tabular}{lllll}
\hline & Latente & Pequeña (NR) & Grande (NR) & Reproductiva \\
\hline Latente & 0.50 & 0.03 & 0.04 & 5.00 \\
Pequeña (NR) & 0.40 & 0.45 & 0.04 & 0.00 \\
Grande (NR) & 0.00 & 0.24 & 0.37 & 0.16 \\
Reproductiva & 0.10 & 0.06 & 0.52 & 0.80 \\
\hline
\end{tabular}

distribución normal $N\left(\mu_{i, j}, \sigma\right)$. El vector $\Delta$ representa el flujo neto de inmigrantes de cada estadio al parche con coordenadas (lon, lat). Con $\Delta_{k, r} N_{\text {lon,lat }}^{i}$ se denota a los inmigrantes del estadio $i$ al parche (lon, lat) provenientes del parche $(k, r)$; es decir, $\Delta_{k, r} N_{\text {lon,lat }}^{i}=\Delta_{k, r}^{\text {lon lat }} N_{k, r}^{i}-\Delta_{\text {lon,lat }}^{k, r} N_{\text {lon,lat }}^{i}$, donde $\Delta_{\gamma}^{\nu} N_{\gamma}^{i}$ es el número de individuos de la clase $i$ que migran del parche $\gamma$ al parche $v$; por tanto la inmigración neta $\Delta$ está dada por:

$$
\begin{aligned}
& \Delta(\text { lon, lat }) \\
& =\left(\sum_{k, r} \Delta_{k, r} N_{\text {lon }, \text { lat }}^{1}, \sum_{k, r} \Delta_{k, r} N_{\text {lon }, \text { lat }}^{2}, \ldots, \sum_{k, r} \Delta_{k, r} N_{\text {lon,lat }}^{n}\right) .
\end{aligned}
$$

$\boldsymbol{S}(x, y)$ es una matriz diagonal de $n \times n$ dimensiones con las idoneidades de cada estadio (ecuación 5). Las variables $x, y$ dependen de la posición (lon, lat), por lo tanto,

$$
\begin{aligned}
& \mathrm{S}(x(\text { lon, lat }), y(\text { lon, lat })) \\
& \quad\left(\begin{array}{ccccc}
S_{\text {lon,lat }}^{1} & \cdot & \cdot & \cdot & 0 \\
0 & S_{\text {lon,lat }}^{2} & \cdot & \cdot & 0 \\
\cdot & \cdot & \cdot & \cdot & \cdot \\
\cdot & \cdot & . & \cdot & \cdot \\
0 & 0 & 0 & 0 & S_{\text {lon,lat }}^{n}
\end{array}\right) .
\end{aligned}
$$

De la estructura del modelo (ecuación 3) se sigue que hay un valor umbral mínimo de idoneidad $S_{0}$ por debajo del cual la supervivencia de la especie es nula; es decir, en ausencia de dispersión, si $S^{i}(x, y) \leq S_{0}$ para todo $i=1, \ldots, n$ entonces la población en $(x, y)$ tiende a extinguirse debido a que su tasa intrínseca de crecimiento $\lambda$ será menor a 1 . El valor $S_{0}$ se calcula como el inverso de la tasa máxima intrínseca de crecimiento

$S_{0}=\frac{1}{\lambda_{\max }}$,

Nótese que el valor máximo de la tasa intrínseca de crecimiento $\lambda_{\max }$ se alcanza en el valor de idoneidad $S(\bar{x}, \bar{y})=1$.

\section{Parametrización del modelo híbrido}

La matriz M que se utilizará en las simulaciones es la correspondiente a la especie Astragalus scaphoides (tabla 1) tomada de la base de datos demográficos COMPADRE (COMPADRE Plant Matrix Database, 2015). Los estadios son latente, pequeña, grande y reproductiva. Para la dispersión se considerarán dos 


\begin{tabular}{|c|c|c|}
\hline 0,05 & 0,2 & 0,05 \\
\hline 0,2 & Init Pos & 0,2 \\
\hline 0,05 & 0,2 & 0,05 \\
\hline
\end{tabular}



Figura 1. Kerneles de dispersión. Imagen izquierda, dispersión a las celdas contiguas (SDD). Imagen derecha, dispersión a celdas distantes (LDD) como función de la distancia $d$ al parche inicial S.

kernels de dispersión que indican el flujo neto de inmigrantes (esto corresponde al término $\Delta$ de las ecuaciones 3 y 4). El primer kernel se refiere a una dispersión a celdas cercanas (kernel SDD) y el segundo a la dispersión a celdas lejanas (kernel LDD). El porcentaje de la población de un parche que se dispersa a los parches contiguos SDD, lo denotamos con $\alpha$ y la que se dispersa a los más lejanos LDD, lo denotamos con $\beta$. Se considera que la dispersión ocurre solo en el estadio de semilla. El kernel SDD se da entre las 8 celdas contiguas a cada parche, con una proporción de $0.2 \alpha$ colonizadores hacia cada una de las 4 celdas laterales y una proporción de $0.05 \alpha$ hacia cada celda diagonal (fig. 1a). La dispersión a celdas no contiguas está determinada por un kernel (LDD) de dispersión exponencial del tipo siguiente:

$P(d)=a \exp \left[-d^{\frac{c}{b}}\right] \quad$ con $a, b, c \in[0,1], x \leq D_{\max }$,

donde $D_{\max }$ es la distancia máxima a la que puede viajar un individuo y $P(d)$ es la fracción de migrantes que viajan a una distancia $d$. La proporción máxima de la población que se desplaza está determinada por $a$. La razón entre $c$ y $b$ define que tan grande es la proporción de individuos que se desplazan a una distancia $d$. Los valores de los parámetros elegidos fueron $a=0.8, b=0.8, c=0.1$ y $D=2$. La distribución del kernel dados estos parámetros se muestra en la figura 1.

Para definir el nicho fundamental de la especie virtual, se eligieron como variables ambientales la temperatura media anual y la precipitación en el mes más húmedo, por ser las más importantes para el desarrollo y supervivencia de varias especies (Bartlein, Prentice y Webb III, 1986; Woodward, 1987). Para analizar el proceso de dispersión se eligió como «espacio geográfico» una zona al noroeste de México. En este espacio la especie virtual inicia el proceso de dispersión, y se definió tomando como base el nicho de la especie, es decir los sitios del espacio geográfico donde la especie puede subsistir. Los valores de temperatura media anual (bio1) y de precipitación en el mes más húmedo (bio13) fueron obtenidos de la página de Worldclim (Hijmans, Cameron, Parra, Jones y Jarvis, 2005) a una resolución de 30 segundos de arco. Aquí suponemos que la tasa óptima de reproducción de la especie virtual se alcanza en las medias $\left(\mu_{x}, \mu_{y}\right)$ de bio1 y bio13 en el espacio geográfico, los cuales fueron $24.2^{\circ} \mathrm{C}$ y $260 \mathrm{~mm}$ (este es el centroide del nicho). La correlación de las variables nichos fue de $\rho=-0.77$ con una desviación de $\sigma_{t}=3.89$ para la bio1 y $\sigma_{P}=9.85$ para bio13. El valor de la tasa máxima intríseca de crecimiento $\left(\lambda_{\max }\right)$ de la matriz $\mathbf{M}$ en condiciones de idoneidad máxima $S\left(\mu_{x}, \mu_{y}\right)=1$ es de $\lambda_{\max }=1.6$

Utilizando la función rasterToPoints del paquete «raster» de R (Hijmans, 2015), se extrajeron los valores de bio1 y bio13 para el espacio geográfico en cuestión, y se aplicaron las ecuaciones (1) y (2) para obtener la idoneidad $S(x, y)$ en cada una de las celdas (fig. 2). Se eligieron aleatoriamente 10 celdas cuya idoneidad del hábitat fuera mayor al valor de $S_{0}$ (en este caso $S_{0}=0.625$ ). En estas celdas se colocaron las poblaciones iniciales de la especie virtual y constituyeron el área inicial de distribución (puntos negros en el espacio geográfico, fig. 3).

\section{Los escenarios}

Se puso a prueba la hipótesis del centroide del nicho (la mayor abundancia se encuentran más cercanos al centroide del nicho)



Figura 2. Nicho fundamental de la especie virtual. La función de distribución $f(x, y)$ que se utilizó para definir al nicho fundamental de la especie virtual, fue la función multinormal bivariada; se eligió como variable $x$ a la temperatura media anual (bio1) y como variable y a la precipitación en el mes más húmedo (bio13). 




Figura 3. Mapa de idoneidad del hábitat. Los puntos negros muestran los sitios donde se colocaron a las 10 poblaciones iniciales de la especie virtual.

bajo distintos escenarios de dispersión: a) sin dispersión de la especie, i.e. $\alpha=0=\beta ; b$ ) con dispersión; se simularon un total de 36 escenarios de dispersión, los cuales son resultado de combinar diferentes proporciones de $\alpha$ y $\beta$. Los valores posibles de $\alpha$ y $\beta$ son $[0.01,0.04,0.06,0.8,0.11,0.14]$. Para realizar las simulaciones se utilizó el paquete demoniche de R (Nenzén et al., 2012).

Se realizaron regresiones lineales entre el índice de idoneidad y la abundancia poblacional, usando los coeficientes de determinación. La comparación de las $r^{2}$ permitió comparar los resultados entre los diferentes escenarios de dispersión.

\section{Resultados}

\section{Modelo sin dispersión}

Las mayores abundancias se encontraron en los valores de idoneidad de hábitat $S$ cercanos al óptimo de la especie (i.e. centroide del nicho). La correlación $r$ fue positiva y la $r^{2}$ fue de 0.95 (fig. 4). La variación no explicada se debe a la estocasticidad introducida en los parámetros demográficos del modelo.

\section{Modelo con dispersión}

En general, los valores que tomó la abundancia fueron más altos conforme la proporción de individuos con dispersión a celdas cercanas SDD fue mayor. Por ejemplo, el número máximo de individuos que alcanzó un parche en el escenario LDD $=0.01$ $\mathrm{SDD}=0.01$ fue de 3,500 individuos, mientras que en el escenario de mayor dispersión ( $\mathrm{LDD}=0.01 \mathrm{SDD}=0.08$ fue de 120,000 individuos (fig. 5).

La relación idoneidad del hábitat $S$ y abundancia fue positiva para todos los escenarios. Sin embargo, se observó que conforme incrementó la proporción de $\alpha$ individuos que viajaban a distancias cortas (SDD), los coeficientes de determinación $r^{2}$ resultaron considerablemente más bajos (fig. 6). En estos escenarios, conforme incrementó la dispersión, la relación entre abundancia e idoneidad se hace más débil, por lo tanto fue posible encontrar abundancias altas en sitios con idoneidad baja (fig. 6).

Las relaciones que se encontraron entre la idoneidad del hábitat y la abundancia poblacional para el escenario donde la proporción de individuos susceptibles a dispersarse a las largas distancias es de $0.01 \leq \beta=0.06$, con proporciones de $0.01 \leq \alpha \leq 0.14$ individuos susceptibles dispersarse a cortas distancias, muestran una correlación $r$ positiva en todos los casos,
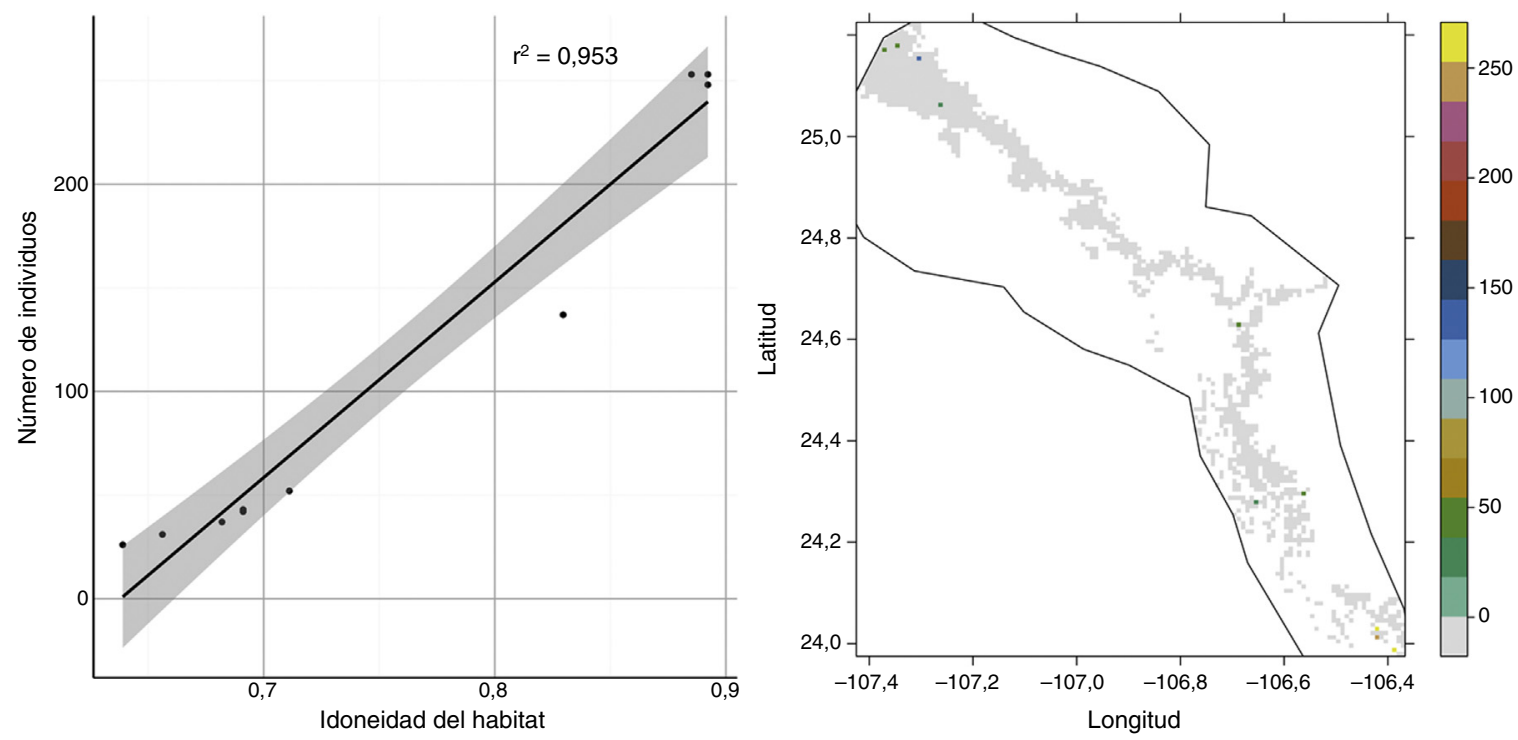

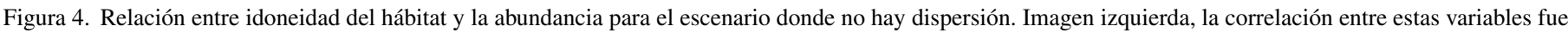

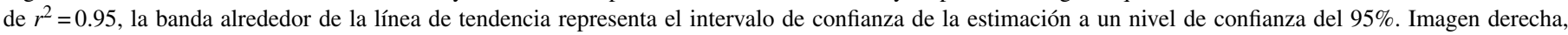
proyección de las abundancias en el espacio geográfico. 

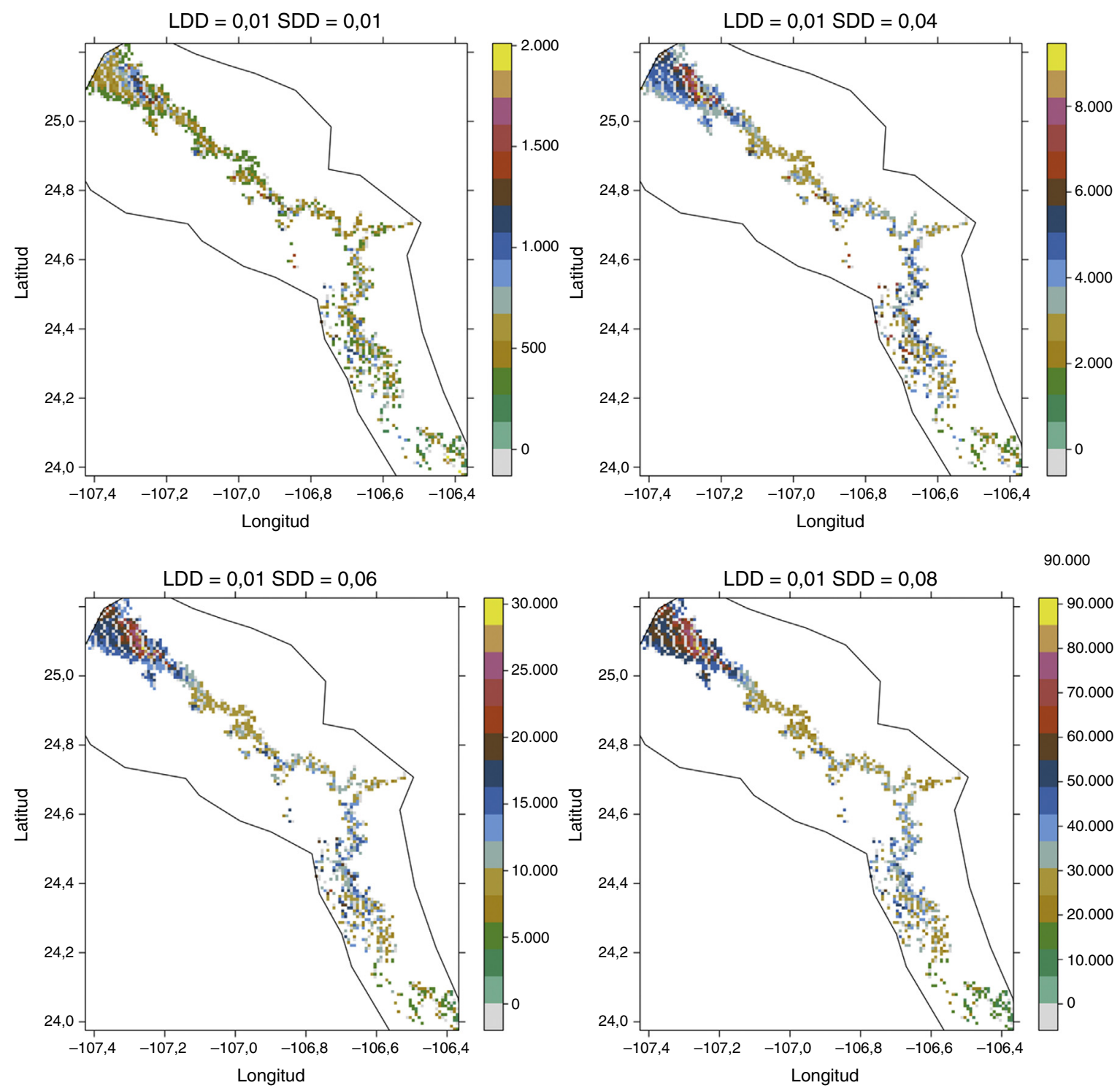

Figura 5. Proyección al espacio geográfico de las abundancias poblacionales para diferentes escenarios de dispersión a cortas distancias SDD ( $0.01 \leq \alpha \leq 0.08)$ y valores de dispersión a largas distancias LDD fijos $(\beta=0.01)$.

sin embargo también muestran que tanto $r$ como $r^{2}$ disminuyen al incrementar las proporciones $\alpha$ y $\beta$ (fig. 6).

En términos generales las $r^{2}$ obtenidas en las simulaciones para todos los escenarios de dispersión disminuyeron conforme se incrementaron las proporciones de individuos con dispersión a celdas cercanas $\operatorname{SDD}(\alpha)$ y de individuos dispersión a celdas no contiguas $\operatorname{LDD}(\beta)$ (fig. 7).

\section{Discusión}

En este trabajo analizamos la relación entre la abundancia de una especie virtual y su posición en el espacio de nicho, utilizando un modelo «híbrido» con estructura de edades que integra procesos demográficos y de dispersión. A pesar de que en la literatura hay trabajos en los que se utilizan modelos híbridos y mecanicistas para estudiar la distribución y abundancia de las especies, la diferencia principal radica en que el nuestro integra diferentes escenarios de dispersión.

El principal resultado que encontramos es que existe una correlación positiva entre abundancia poblacional y la idoneidad de hábitat, es decir, se confirma la hipótesis del centroide del nicho. Otro resultado fundamental es que esta correlación está regulada por la relación que guardan los parámetros demográficos respecto a los de la dispersión.

Los resultados obtenidos muestran que la intensidad de la correlación positiva entre abundancia e idoneidad del hábitat depende de la capacidad de dispersión de la especie en cuestión: la correlación entre abundancia e idoneidad del hábitat es casi perfecta en un escenario de no dispersión; sin embargo, cuando se incluye el factor dispersión, la correlación declina abruptamente pudiéndose encontrar poblaciones con abundancias mayores en sitios donde la calidad del hábitat es baja. Este resultado concuerda con lo señalado por Pulliam (2000), quien 

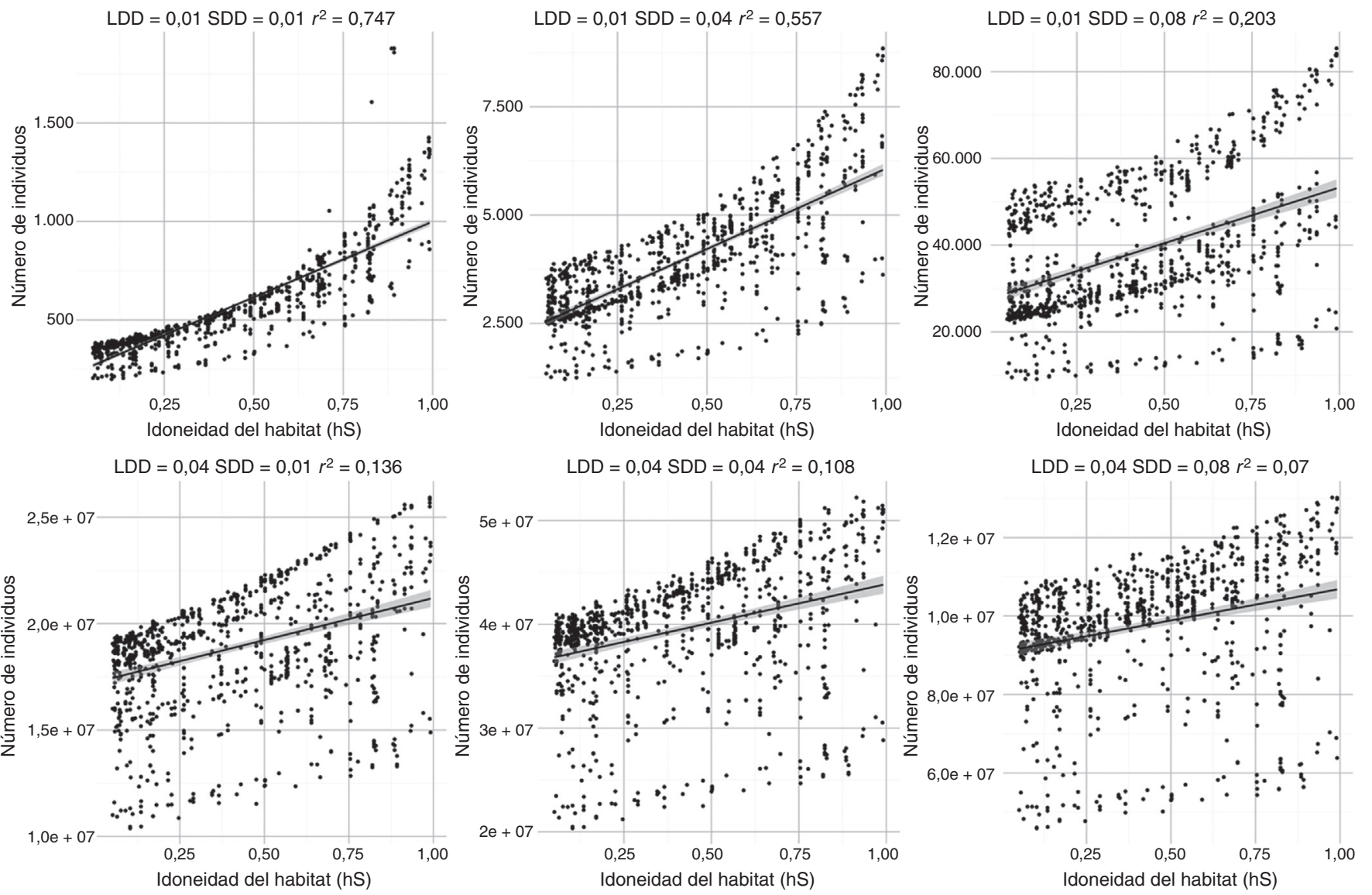

$\operatorname{LDD}=0,08 \mathrm{SDD}=0,01 r^{2}=0,004$

$\operatorname{LDD}=0,08 \mathrm{SDD}=0,04 r^{2}=0,003$
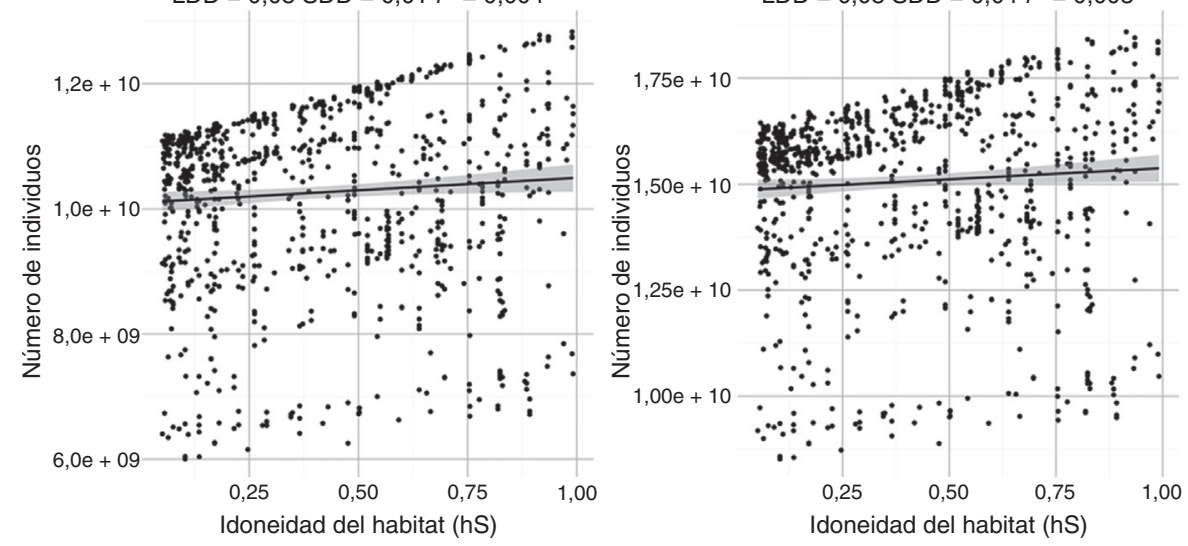

$\operatorname{LDD}=0,08 \mathrm{SDD}=0,08 r^{2}=0,002$

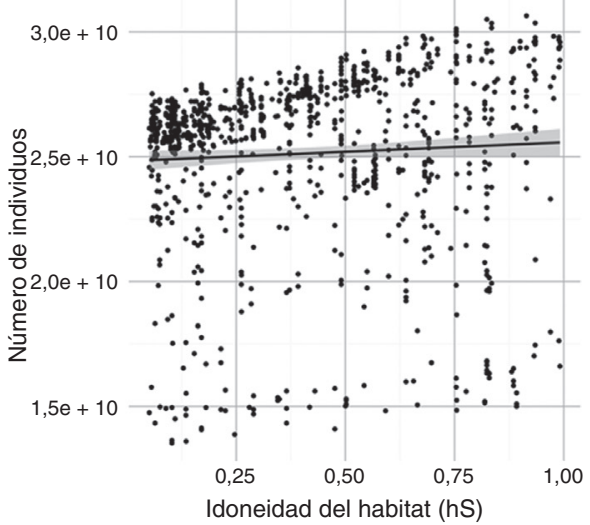

Figura 6. Correlaciones entre idoneidad del hábitat y las abundancias poblacionales para diferentes escenarios de dispersión a cortas distancias SDD y a largas distancias LDD. La banda alrededor de las líneas de tendencia representa el intervalo de confianza de la estimación a un nivel de confianza del $95 \%$.

afirmó que las especies frecuentemente se encuentran en sitios donde su tasa de crecimiento es negativa (Pulliam, 2000; Schurr et al., 2012). A este fenómeno se le ha denominado dinámica fuente-sumidero (Pagel y Schurr, 2012; Pulliam, 1988, 2000; Schurr et al., 2012) y depende en gran medida de si las respuestas densodependientes afectan la dinámica de dispersión de la especie.

Nuestros resultados refuerzan las predicciones teóricas informales derivadas de la teoría del nicho de Hutchinson (Maguire, 1973; Schurr et al., 2012) y da elementos teóricos a los resultados empíricos como los de VanDerWal et al. (2009), Escalante y Martínez-Meyer (2013) y Martínez-Meyer et al. (2013), ya que dan una explicación plausible en términos poblacionales a las correlaciones que se obtienen entre abundancia y distancia en el espacio de nicho. Además, sugieren algunas hipótesis acerca de las condiciones en las que los procesos demográficos y de dispersión pueden hacer que la correlación entre abundancia y distancia al centroide del nicho sea baja, por ejemplo, se espera que las especies cuyas tasas de migración son altas, muestren una correlación baja entre su abundancia y su nicho.

Asimismo, nuestro trabajo complementa estudios como el de Holt, Lawton, Gaston y Blanchette (1997) en el que se analiza la relación entre la abundancia y el rango de distribución de la especie. A diferencia del enfoque de Holt et al., que se basa en la 


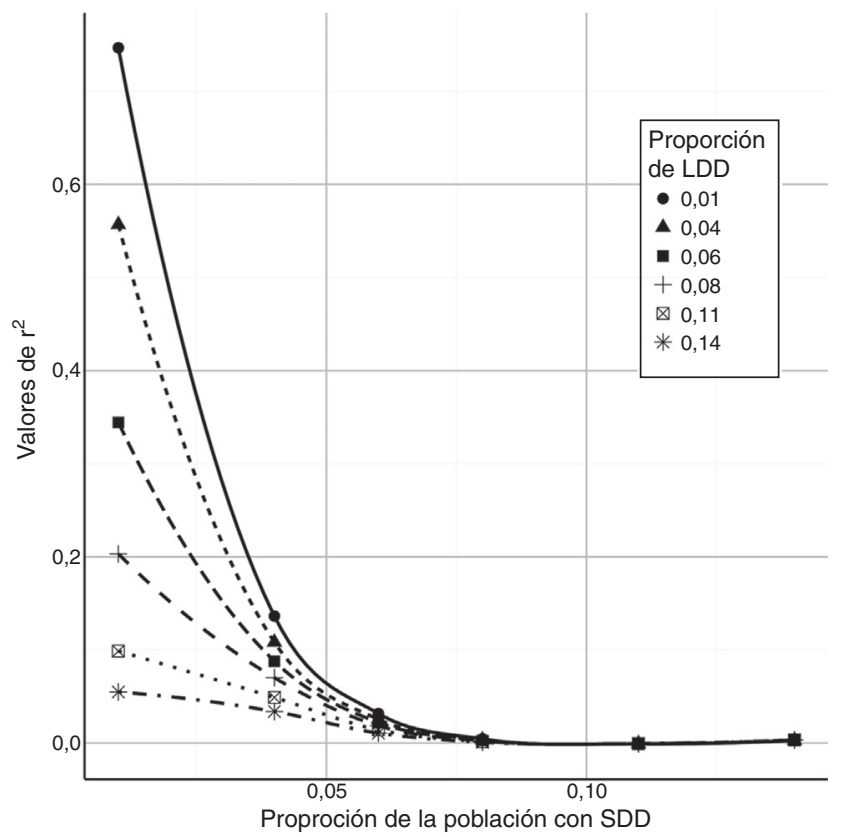

Figura 7. Valores de $r^{2}$ para todos los escenarios de dispersión a grandes y a cortas distancias.

idea de que el proceso de dispersión de la especie ha concluido y no consideran el efecto de la migración-inmigración sobre las abundancias locales, nosotros mostramos cómo el proceso de dispersión y el nicho pueden determinar la abundancia de las poblaciones en el rango geográfico.

Finalmente, si bien el enfoque de los modelos híbridos es prometedor, existe una limitante práctica para incluir el término de dispersión de estos modelos, ya que la estimación de los kernels de dispersión es difícil, los datos son escasos y kerneles con colas largas son matemáticamente difíciles de manejar (Barve et al., 2011). A pesar de que en este trabajo se enfatizó el papel de la dispersión en la relación entre abundancia y posición en el espacio de nicho, otra limitante importante para la comprensión de esta relación son las interacciones, de manera que la ecuación de abundancia de una especie en un espacio geográficamente explícito debe ser una función de su nicho fundamental, su capacidad de dispersión y las interacciones que tenga con las demás especies (Soberón, 2010). El trabajo que presentamos aquí pretende ser una base teórica sobre la cual avanzar incluyendo este otro tipo de factores, lo cual permitirá entender mejor la compleja red de factores que determinan la geometría y la estructura interna del área de distribución de una especie.

\section{Agradecimientos}

Se agradece al Posgrado en Ciencias Biológicas por la formación académica de Luis Osorio. Asimismo se agradece al PAPIIT-UNAM IN112715 (2015) por el apoyo parcial brindado para la realización de esta investigación. Jorge Soberón agradece al proyecto NSF 1208472. Agradecemos a Andrés Lira-Noriega y a los revisores anónimos cuyos comentarios nos ayudaron a mejorar la presentación del trabajo.

\section{Referencias}

Bartlein, P. J., Prentice, I. C. y Webb, T., III. (1986). Climatic response surfaces from pollen data for some eastern North American Taxa. Journal of Biogeography, 13, 35-57.

Barve, N., Barve, V., Jiménez-Valverde, A., Lira-Noriega, A., Maher, S. P., Peterson, A. T., et al. (2011). The crucial role of the accessible area in ecological niche modeling and species' distribution modeling. Ecological Modelling, 222, 1810-1819.

Broennimann, O., Fitzpatrick, M. C., Pearman, P. B., Petitpierre, B., Pellissier, L., Yoccoz, N. G., et al. (2012). Measuring ecological niche overlap from occurrence and spatial environmental data. Global Ecology and Biogeography, 21, 481-497.

Buckley, L., Urban, M., Angilletta, M., Crozier, L., Rissler, L. y Sears, M. (2010). Can mechanism inform species' distribution models? Ecology Letters, 13, $1041-1054$

Cain, S. A. (1944). Foundations of plant geography. New York: Harper \& Brothers.

Caswell, H. (2000). Matrix population models: construction, analysis and interpretation. Sunderland, Massachusetts: Sinauer Associates.

Catterall, S., Cook, A. R., Marion, G., Butler, A. y Hulme, P. E. (2012). Accounting for uncertainty in colonization times: a novel approach to modelling the spatio-temporal dynamics of alien invasions using distribution data. Ecography, 35, 901-911.

COMPADRE Plant Matrix Database (2015). Max Planck Institute for demographic research (Germany). (consultado 19 Sep 2015) Disponible en: www.compadre-db.org.

De Moraes, M. y Viveiros, C. (2012). Does environmental suitability explain the relative abundance of the tailed tailless bat, Anoura caudifer? Natureza a Conservacao, 10, 221-227.

Dormann, C. F. (2009). Response to comment on methods to account for spatial autocorrelation in the analysis of species' distributional data: a review. Ecography, 32, 379-381.

Escalante, T. y Martínez-Meyer, E. (2013). Ecological niche modeling and wildlife management units (UMAS): an application to deer in campeche, México. Tropical and Subtropical Agroecosystems, 16, 183-191.

Farber, O. y Kadmon, R. (2003). Assessment of alternative approaches for bioclimatic modeling with special emphasis on the Mahalanobis distance. Ecological Modeling, 160, 115-130.

Franklin, J. (2010). Moving beyond static species' distribution models in support of conservation biogeography. Diversity and Distributions, 16, 321-330.

Gallien, L., Münkemüller, T., Albert, C. H., Boulangeat, I. y Thuiller, W. (2010). Predicting potential distributions of invasive species: where to go from here? Diversity and Distributions, 16, 331-342.

Gaston, K. J. (2003). The structure and dynamics of geographic ranges. Oxford series in ecology and evolution. New York: Oxford University Press.

Grinnell, J. (1917). The niche-relationships of the California thrasher. The Auk, $34,427-433$.

Guisan, A. y Thuiller, W. (2005). Predicting species' distribution: offering more than simple habitat models. Ecology Letters, 8, 993-1009.

Helaouet, P., Beaugrand, G. y Edwards, M. (2013). Understanding long-term changes in species' abundance using a niche-based approach. PloS one, 8 , e79186.

Hijmans, R. J. (2015). Raster: geographic data analysis and modeling. R package version 2.4-18. (consultado 4 Nov 2015). Disponible en: http://CRAN.Rproject.org $/$ package $=$ raster.

Hijmans, R. J., Cameron, S. E., Parra, J. L., Jones, P. G. y Jarvis, A. (2005). The WorldClim interpolated global terrestrial climate surfaces. (consultado el 01 Oct 2014). Disponible en: http://www.worldclim.org/.

Holt, R. D., Lawton, J. H., Gaston, K. J. y Blanchette, C. A. (1997). On the relationship between range size and local abundance: back to basics. Oikos, $78,183-190$

Hutchinson, G. E. (1957). Concluding remarks. Cold Spring Harbor Symposia on Quantitative Biology, 22, 415-427.

Jongejans, E., Shea, K., Skarpaas, O., Kelly, D., Sheppard, A. W. y Woodburn, T. L. (2008). Dispersal and demography contributions to population spread of Carduus nutans in its native and invaded ranges. Journal of Ecology, 96, $687-697$. 
Kearny, M. y Porter, W. (2009). Mechanistic niche modelling: combining physiological and spatial data to predict species' ranges. Ecology Letters, 12, 334-350.

Lira-Noriega, A., Soberón, J. y Milller, C. (2013). Process-based and correlative modeling of desert mistletoe distribution: a multiscalar approach. Ecosphere, 4, 1-23.

Maguire, B., Jr. (1973). Niche response structure and the analytical potentials of its relationship to the habitat. The American Naturalist, 107, 213-246.

Marion, G., McInerny, G. J., Pagel, J., Catterall, S., Cook, A. R., Hartig, F., et al. (2012). Parameter and uncertainty estimation for process-oriented population and distribution models: data, statistics and the niche. Journal of Biogeography, 39, 2225-2239.

Martínez-Meyer, E., Díaz-Porras, D., Townsend, P. y Yáñez-Arenas, C. (2013). Ecological niche structure and range wide abundance patterns of species. Biology Letters, 9, 1-5.

Nenzén, H. K., Swab, R. M., Keith, D. A. y Araújo, M. B. (2012). Demoniche - an R-package for simulating spatially-explicit population dynamics. Ecography, 35, 577-580.

Pagel, J. y Schurr, F. M. (2012). Forecasting species' ranges by statistical estimation of ecological niches and spatial population dynamics. Global Ecology and Biogeography, 21, 293-304.

Pulliam, H. R. (1988). Sources, sinks, and population regulation. The American Naturalist, 132, 652-661.

Pulliam, H. R. (2000). On the relationship between niche and distribution. Ecology Letters, 3, 349-361.

Sagarin, D. R., Gaines, S. y Gaylord, B. (2006). Moving beyond assumptions to understand abundance distributions across the ranges of species. Trends in Ecology and Evolution, 21(9), 524-530.

Saupe, E. E., Barve, V., Myers, C. E., Soberón, J., Barve, N., Hensz, C. M., et al. (2012). Variation in niche and distribution model performance: the need for a priori assessment of key causal factors. Ecological Modelling, 237-238, $11-22$.

Schurr, F. M., Midgley, G. F., Rebelo, A. G., Reeves, G., Poschlod, P. y Higgins, S. I. (2007). Colonization and persistence ability explain the extent to which plant species fill their potential range. Global Ecology and Biogeography, 16, 449-459.

Schurr, F. M., Pagel, J., Cabral, J. S., Groeneveld, J., Bykova, O., O’Hara, R. B., et al. (2012). How to understand species' niches and range dynamics: a demographic research agenda for biogeography. Journal of Biogeography, 39, 2146-2162.

Siqueira, T., Bini, L., Cianciaruso, M., Oliveira, F. y Trivinho-Strixino, S. (2009). The role of niche measures in explaining the abundance-distribution relationship in tropical lotic chironomids. Hydrobiologia, 1-10

Soberon, J. (2010). Niche and area of distribution modeling: a population ecology perspective. Ecography, 33, 159-167.

Thuiller, W., Albert, C., Araujo, M. B., Berry, P. M., Cabeza, M., Guisan, A., et al. (2008). Predicting global change impacts on plant species' distributions: future challenges. Perspectives in Plant Ecology Evolution and Systematics, 9, 137-152.

Torres, M. N., Júnior, P. D. M., Santos, T., Silveira, L., Jácomo-de Almeida, A. T. y Diniz-Filho, A. F. (2012). Can species' distribution modelling provide estimates of population densities? A case study with jaguars in the Neotropics. Diversity and Distributions, 18, 615-627.

Udvary, M. D. F. (1969). Dynamic zoogeography: with special reference to land animals. New York: Van Nostrand Reinhold Comp.

VanDerWal, J., Shoo, L., Johnson, C. y Williams, S. (2009). Abundance and the environmental niche: environmental suitability estimated from niche models predicts the upper limit of local abundance. The American Naturalist, 174, 282-291.

Woodward, F. I. (1987). Climate and plant distribution. Cambridge: Cambridge University Press.

Yáñez-Arenas, C., Guevara, R., Martínez-Meyer, E., Mandujano, S. y Lobo, J. M. (2014). Predicting species' abundances from occurrence data: effects of sample size and bias. Ecological Modelling, 294, 36-41.

Yáñez-Arenas, C., Martińez-Meyer, E., Mandujano, S. y Rojas-Soto, O. (2012). Modelling geographic patterns of population density of the white-tailed deer in central Mexico by implementing ecological niche theory. Oikos, 121, 2081-2089. 